\title{
Correlation Adaptive Compressive Sensing of Wireless Sensor Network
}

\author{
Wang Changliang and Fang Jie \\ ZheJiang Industry Polytechnic College, Shaoxing, Zhejiang, 312000, China \\ Wangcl98@163.com
}

\begin{abstract}
Correlation of wireless sensor network data is the foundation and premise of using compressive sensing theory to reconstruct the network data as well as the main basis for the design of network data reconstruction algorithm. However, various interferences of network data in practical transmission would result in consistent changes of correlation, thus lead to sharply aggravation of reconstruction performances in the existing compressive sensing theory. In order to improve the reliability and efficiency of compressive sensing of wireless sensor network data, a correlation adaptive reconstruction algorithm for network data is proposed. This algorithm first estimates the correlation of data to be reconstructed through iteration and then restores the data to be restructured using the two-step correlation test method of support elements. Compared to the previous reconstruction methods, correlation adaptive reconstruction method can greatly improve the reconstruction accuracy when the correlation of network data is changing.
\end{abstract}

Keywords: wireless sensor network, compressive sensing, data reconstruction, correlation; adaption

\section{Introduction}

Wireless Sensor Networks (WSN) has a wide application in such fields as military, transportation, medical treatment and environmental monitoring with advantages of high monitoring accuracy, good fault-tolerance performance and large coverage[1]. However, data sensed by numerous nodes in the WSN is huge; a lot of electrical energy would be consumed in the wireless transmission; meanwhile, the battery capacity carried by network nodes of wireless sensor is limited and it is hard to complement the consumed energy. Therefore, the research hotspot is how to reduce energy consumption and extend the network lifecycle under the premise of guaranteeing monitoring tasks [2].

Recently, a new signal sampling theory with full use of signal sparsity or compressibility- Compressive sensing (CS) gradually develops [3-5]. This theory shows that when the signal is of sparsity and compressibility, the accurate reconstruction of signals can be achieved by collecting a small amount of signal measurements. In the network of wireless sensor, the same case in the same region has been observed by numerous nodes and the data sensed by each node has a strong correlation (compressibility). A small amount of measurements are used to operate the Compressive sensing (CS) and the compression and reconstruction of data can be achieved. Therefore, the transmission quantity of network data can be evidently reduced and the aim of energy-consumption can be achieved.

The essence of reconstructing network data by a small amount of measurements is the solution of linear equations under underdetermined conditions. There are countless solutions for linear equations under underdetermined conditions, so it tends to be converted into the Norm 1 minimization for solution in existing research. Originally, researchers used the Linear Programming (LP) algorithm to solve the Norm 1 
minimization problem, but they got the Basis Pursuit (BP) algorithm[6]. The Basis Pursuit can get higher reconstruction accuracy with extreme high complexity, so data reconstruction can be hardly achieved in terms of large-scale wireless sensor networks under practical conditions. In order to reduce complexity of reconstruction algorithm, researchers proposed various greedy algorithms to solve the Norm 1 minimization problem, among which there are some representative algorithms including Matching Pursuit (MP) [7], Orthogonal Matching Pursuit (OM) [8], Stagewise OMP (StOMP) [9].

Compared to linear programming, greedy algorithm evidently reduces the complexity but reconstruction requires more measurement computing with poor accuracy. In order to overcome the disadvantage of greedy algorithm, researchers again proposed the improved greedy algorithms such as subspace pursuit algorithm [10] and compressive sampling matching pursuit algorithm [11]. These algorithms can achieve similar reconstruction accuracy like linear programming with lower complexity than the latter, which makes large-scale wireless sensor network data reconstruction operational. The reconstruction of above algorithms all require prior correlation information of data to be reconstructed. However, the practical network data transmission is inevitably affected by various interferences, thus leading to consistent changes of network data correlation. On the one hand, it may make the compressive sensing reconstruction algorithm fail; on the other hand, it may increase the complexity of compressive sensing measurement redundancy and reconstruction algorithm. Therefore, in terms of network data with changing correlation, traditional data reconstruction algorithm all cannot achieve the optimal performance.

\section{Descriptions of Problems}

In the WSN model shown in Figure 1, if $N$ sensor nodes are randomly scattered in the perceived area, they will constitute a wireless network through self-organization, and collect and process the specific information in the area covered by the network by means of collaboration. Considering that various nodes collect data simultaneously when WSN is working, we use $x_{n}(n \in\{1,2, \ldots, N\})$ to represent the data perceived by nth node. Before sending data, each node produces a group of pseudo-random numbers locally and forms a corresponding pseudo-random sequence, which is represented by $r_{n}(m)$, where $m=1,2, \ldots, M \quad(M \ll N)$. Equipped with wireless communication devices, each sensor node can send the perceived data to Sink node. When sending data, various nodes shall multiply data $x_{n}$ and the local random sequence element, and thus get $N$ :

$$
y(m)=\sum_{n=1}^{N} x_{n} r_{n}(m)
$$

And then transmit them to the Sink node. After multiplying various nodes and the local random sequence element and sending the corresponding product, we can get a measurement vector composed of $M$ measurements, namely, $y=[y(1), y(2), \ldots, y(M)]^{T}$. If we regard the pseudo-random sequence generated by various nodes as row of matrix, the pseudo-random sequence generated by $N$ nodes can form the following $M \times N$-dimensional matrix:

$$
\Phi=\left[\begin{array}{ccccc}
r_{1}(1) & r_{2}(1) & \cdots & r_{N-1}(1) & r_{N}(1) \\
r_{1}(2) & r_{2}(2) & \cdots & r_{N-1}(2) & r_{N}(2) \\
\vdots & \vdots & \cdots & \vdots & \vdots \\
r_{1}(J) & r_{2}(J) & \cdots & r_{N-1}(M) & r_{N}(M)
\end{array}\right]_{M \times N}
$$

Therefore, the relationship between the data received in Sink node and the original data collected in sensor nodes can be expressed as follows: 


$$
\mathbf{y}=\mathbf{\Phi} \mathbf{x}
$$

Where $\mathbf{x}=\left[x_{1}, x_{2}, \ldots, x_{N}\right]^{T}$. We may notice that the process of reconstructing the original data based on Formula (2) can be seen as typical sparse signal reconstruction issue in compressive sensing theory. As $M \ll N$, the linear equation with $\mathbf{x}$ as solution is in an undetermined status, namely, the equation has numerous solutions. The previous network data reconstruction method used to convert the issue of solving equation into the issue of minimizing norm 1 :

$$
\hat{\mathbf{x}}=\arg \min \|\mathbf{x}\|_{1} \text { s.t. } \quad \mathbf{y}=\boldsymbol{\Phi} \mathbf{x}
$$

\section{Proposed Algorithm}

In order to verify the correctness and effectiveness of the algorithm, theoretical deviation is conducted for the convergence of the proposed algorithm. It can be seen from the algorithm steps in Section 2 that in order to prove the convergence of algorithm, we only need prove that residual error $\mathbf{r}_{1}=\mathbf{y}-\boldsymbol{\Phi}_{\mathbf{F}_{1}}\left(\boldsymbol{\Phi}_{\mathbf{F}_{1}}^{\mathrm{T}} \boldsymbol{\Phi}_{\mathbf{F}_{1}}\right)^{-1} \boldsymbol{\Phi}_{\mathbf{F}_{1}}^{\mathrm{T}} \cdot \mathbf{y}$ decreases progressively in each iterative process. Lemmas are given as follows:

Lemma 1 As for different integers $K_{1}$ and $K_{2}$, if $K_{1} \leq K_{2}$, restricted isometry constant $\delta$ meets:

$$
\delta_{K_{1}} \leq \delta_{K_{2}}
$$

Proof: Assuming that $\boldsymbol{\Phi}$ meets the limited isometry property, as for any vector $\mathbf{q}_{1} \in R^{K_{1}}$, it can be extended to be $\mathbf{q}_{2} \in R^{K_{2}}$ through supplementing $K_{2}-K_{1}$ zero values. Based on the definition of isometry property, $J \subset\{1, \cdots, N\}$ and $|J| \leq K_{2}$, and then

$$
\left(1-\delta_{K_{2}}\right)\left\|\mathbf{q}_{2}\right\|_{2}^{2} \leq\left\|\mathbf{\Phi}_{\mathrm{J}} \mathbf{q}_{2}\right\|_{2}^{2} \leq\left(1-\delta_{K_{2}}\right)\left\|\mathbf{q}_{2}\right\|_{2}^{2}
$$

is established. If $|I| \leq K_{1}$,

$$
\left(1-\delta_{K_{2}}\right)\left\|\mathbf{q}_{1}\right\|_{2}^{2} \leq\left\|\mathbf{\Phi}_{1} \mathbf{q}_{1}\right\|_{2}^{2} \leq\left(1-\delta_{K_{2}}\right)\left\|\mathbf{q}_{1}\right\|_{2}^{2}
$$

When defining $\delta_{K_{1}}$ as the minimum $\delta$ value meeting Formula (5), and then

$$
\delta_{K_{1}} \leq \delta_{K_{2}}
$$

And thus the conclusions are proved.

Lemma 2: If set $I, J \subset\{1, \cdots, N\}$ is established, $I \cap J=\phi$; assuming that $\delta_{|I|+|J|}<1$, the following is established for any vectors $\mathbf{a} \in R^{|I|}$ and $\mathbf{b} \in R^{|J|}$

$$
\left|\left\langle\mathbf{\Phi}_{\mathbf{I}} \mathbf{a}, \boldsymbol{\Phi}_{\mathbf{J}} \mathbf{b}\right\rangle\right| \leq \delta_{|I|+|J|}\|\mathbf{a}\|_{2}\|\mathbf{b}\|_{2}
$$

Proof: If any one of $\|\mathbf{a}\|_{2}$ or $\|\mathbf{b}\|_{2}$ is zero, the conclusion is established. If both $\|\mathbf{a}\|_{2}$ and $\|\mathbf{b}\|_{2}$ are not zero and the following formula is established

$$
\mathbf{a}^{\prime}=\frac{\mathbf{a}}{\|\mathbf{a}\|_{2}}, \quad \mathbf{b}^{\prime}=\frac{\mathbf{b}}{\|\mathbf{b}\|_{2}}, \quad \mathbf{x}^{\prime}=\Phi_{\mathrm{I}} \mathbf{a}, \quad \mathbf{y}^{\prime}=\Phi_{\mathrm{J}} \mathbf{b}
$$

The following formula can be established based on the definition of restricted isometry property 


$$
\begin{aligned}
& 2\left(1-\delta_{|I|+|J|}\right) \leq\left\|\mathbf{x}^{\prime}+\mathbf{y}^{\prime}\right\|_{2}^{2} \\
& =\|\left[\boldsymbol{\Phi}_{\mathbf{i}} \boldsymbol{\Phi}_{\mathbf{j}}\left[\begin{array}{l}
\mathbf{a}^{\prime} \\
\mathbf{b}^{\prime}
\end{array}\right] \|_{2}^{2} \leq 2\left(1+\delta_{|I|+|J|}\right)\right. \\
& 2\left(1-\delta_{|I|+|J|}\right) \leq\left\|\mathbf{x}^{\prime}-\mathbf{y}^{\prime}\right\|_{2}^{2} \\
& =\left\|\left[\begin{array}{|}
\boldsymbol{\Phi}_{\mathbf{i}} \boldsymbol{\Phi}_{j} \\
j
\end{array}\right]\left[\begin{array}{c}
\mathbf{a}^{\prime} \\
-\mathbf{b}^{\prime}
\end{array}\right]\right\|_{2}^{2} \leq 2\left(1+\delta_{|I|+|J|}\right)
\end{aligned}
$$

And then the following formulas are established

$$
\begin{aligned}
\left\langle\mathbf{x}^{\prime}, \mathbf{y}^{\prime}\right\rangle & =\frac{\left\|\mathbf{x}^{\prime}+\mathbf{y}^{\prime}\right\|_{2}^{2}-\left\|\mathbf{x}^{\prime}-\mathbf{y}^{\prime}\right\|_{2}^{2}}{4} \leq \delta_{|I|+|J|} \\
-\left\langle\mathbf{x}^{\prime}, \mathbf{y}^{\prime}\right\rangle & =\frac{\left\|\mathbf{x}^{\prime}-\mathbf{y}^{\prime}\right\|_{2}^{2}-\left\|\mathbf{x}^{\prime}+\mathbf{y}^{\prime}\right\|_{2}^{2}}{4} \leq \delta_{|I|+|J|}
\end{aligned}
$$

And then

$$
\frac{\left|\left\langle\boldsymbol{\Phi}_{\mathbf{I}} \mathbf{a}, \boldsymbol{\Phi}_{\mathrm{J}} \mathbf{b}\right\rangle\right|}{\|\mathbf{a}\|_{2}\|\mathbf{b}\|_{2}}=\left|\left\langle\mathbf{x}^{\prime}, \mathbf{y}^{\prime}\right\rangle\right| \leq \delta_{|I|+|J|}
$$

The conclusions are proved.

Lemma 3: As for matrix $\boldsymbol{\Phi} \in R^{M \times N}$ and set $I, J \subset\{1, \cdots, N\}, I \cap J=\phi$, if $\delta_{|I|+|J|}<1$, $\mathbf{y} \in \operatorname{span}\left(\boldsymbol{\Phi}_{\mathbf{I}}\right), \mathbf{r}=\mathbf{y}-\boldsymbol{\Phi}_{\mathbf{J}}\left(\boldsymbol{\Phi}_{\mathbf{J}}^{\mathrm{T}} \boldsymbol{\Phi}_{\mathbf{J}}\right)^{-1} \boldsymbol{\Phi}_{\mathbf{J}}^{\mathrm{T}} \cdot \mathbf{y}$, and then

$$
\left(1-\frac{\delta_{|I|+|J|}}{1-\delta_{\text {max }}(|I|,|J|)}\right)\|\mathbf{y}\|_{2} \leq\|\mathbf{r}\|_{2} \leq\|\mathbf{y}\|_{2}
$$

Proof: If $\mathbf{y}_{\mathrm{p}}=\boldsymbol{\Phi}_{\mathrm{J}} \mathbf{x}_{\mathrm{p}}, \mathbf{y}=\boldsymbol{\Phi}_{\mathrm{I}} \mathbf{x}$, the following formulas can be established based on Lemma 2

And then

$$
\begin{array}{r}
\left|\left\langle\mathbf{y}_{\mathbf{p}}, \mathbf{y}\right\rangle\right|=\left|\left\langle\mathbf{\Phi}_{\mathbf{J}} \mathbf{x}_{\mathbf{p}}, \boldsymbol{\Phi}_{\mathbf{I}} \mathbf{x}\right\rangle\right| \\
\leq \delta_{|I|+|J|}\left\|\mathbf{x}_{\mathbf{p}}\right\|_{2}\|\mathbf{x}\|_{2} \\
\leq \delta_{|I|+|J|} \frac{\left\|\mathbf{y}_{\mathbf{p}}\right\|_{2}}{\sqrt{1-\delta_{|J|}}} \frac{\| \mathbf{y}_{2}}{\sqrt{1-\delta_{|I|}}} \\
\leq \frac{\delta_{|I|+|J|}}{1-\delta_{\max (|I||| J \mid)}}\left\|\mathbf{y}_{\mathbf{p}}\right\|_{2}\|\mathbf{y}\|_{2}
\end{array}
$$

$$
\text { Meanwhile }\left\langle\mathbf{y}_{\mathrm{p}}, \mathbf{y}\right\rangle=\left\langle\mathbf{y}_{\mathrm{p}}, \mathbf{y}_{\mathrm{p}}+\mathbf{r}\right\rangle=\left\|\mathbf{y}_{\mathbf{p}}\right\|_{2}^{2}
$$

$$
\left\|\mathbf{y}_{\mathbf{p}}\right\|_{2} \leq \frac{\delta_{|I|+|J|}}{1-\delta_{\text {max }}(|I|,|J|)}\|\mathbf{y}\|_{2}
$$

The following formula is established based on triangle inequality

$$
\|\mathbf{r}\|_{2}=\left\|\mathbf{y}-\mathbf{y}_{\mathbf{p}}\right\|_{2} \geq\|\mathbf{y}\|_{2}-\left\|\mathbf{y}_{\mathbf{p}}\right\|_{2}
$$

And then 
Because

$$
\|\mathbf{r}\|_{2} \geq\left(1-\frac{\delta_{|I|+|J|}}{1-\delta_{\text {max }}(|I|,|J|)}\right)\|\mathbf{y}\|_{2}
$$

The conclusions are proved.

$$
\|\mathbf{r}\|_{2}^{2}+\left\|\mathbf{y}_{\mathbf{p}}\right\|_{2}^{2}=\|\mathbf{y}\|_{2}^{2}
$$

Theorem: Assuming that the sparsity of vector $x \in R^{N}$ to be reconstructed is $K$ and $\mathbf{y}=\boldsymbol{\Phi \mathbf { x }}\left(y \in R^{M}\right)$ is established, if the measurement matrix meets the restricted isometry property and its restricted isometry constant meets $\delta_{3 K}<0.165$, residual error $\mathbf{r}$ definite based on $\mathbf{r}_{1}=\mathbf{y}-\boldsymbol{\Phi}_{\mathrm{F}_{1}}\left(\boldsymbol{\Phi}_{\mathrm{F}_{1}}^{\mathrm{T}} \boldsymbol{\Phi}_{\mathrm{F}_{1}}\right)^{-1} \boldsymbol{\Phi}_{\mathrm{F}_{1}}^{\mathrm{T}} \cdot \mathbf{y}$ meet

$$
\left\|\mathbf{r}_{1}\right\|_{2} \leq \frac{c_{K}}{1-2 \delta_{3 K}}\left\|\mathbf{r}_{1-1}\right\|_{2}<\left\|\mathbf{r}_{1-1}\right\|_{2}
$$

Where $c_{K}=\frac{2 \delta_{3 K}\left(1+\delta_{3 K}\right)}{\left(1-2 \delta_{3 K}\right)^{3}}$.

Proof: Taking $\mathbf{x}_{\mathbf{F}-\mathbf{F}_{1}}$ as the residual error of signal vector after $l$ th iteration, the corresponding measurement vector is $\boldsymbol{\Phi}_{\mathbf{F}-\mathbf{F}_{1}}$, and the residual error of measurement can be written as

$$
\begin{aligned}
\mathbf{r}_{1} & =\mathbf{y}-\boldsymbol{\Phi}_{F_{1}}\left(\Phi_{F_{1}}^{\mathrm{T}} \boldsymbol{\Phi}_{\mathrm{F}_{1}}\right)^{-1} \boldsymbol{\Phi}_{\mathrm{F}_{1}}^{\mathrm{T}} \cdot \mathbf{y} \\
& =\left(\boldsymbol{\Phi}_{\mathrm{F}-\mathrm{F}_{1}} \mathbf{x}_{\mathrm{F}-\mathrm{F}_{1}}+\boldsymbol{\Phi}_{\mathrm{F}_{1}} \mathbf{X}_{\mathrm{F}_{1}}\right)-\boldsymbol{\Phi}_{\mathrm{F}_{1}}\left(\Phi_{\mathrm{F}_{1}}^{\mathrm{T}} \boldsymbol{\Phi}_{\mathrm{F}_{1}}\right)^{-1} \boldsymbol{\Phi}_{\mathrm{F}_{1}}^{\mathrm{T}} \cdot\left(\boldsymbol{\Phi}_{\mathrm{F}-\mathrm{F}_{1}} \mathbf{X}_{\mathrm{F}-\mathrm{F}_{1}}+\boldsymbol{\Phi}_{\mathrm{F}_{1}} \mathbf{X}_{\mathrm{F}_{1}}\right) \\
& =\boldsymbol{\Phi}_{\mathrm{F}-\mathrm{F}_{1}} \mathbf{x}_{\mathrm{F}-\mathrm{F}_{1}}-\boldsymbol{\Phi}_{\mathrm{F}_{1}}\left(\boldsymbol{\Phi}_{\mathrm{F}_{1}}^{\mathrm{T}} \boldsymbol{\Phi}_{\mathrm{F}_{1}}\right)^{-1} \boldsymbol{\Phi}_{\mathrm{F}_{1}}^{\mathrm{T}} \cdot \boldsymbol{\Phi}_{\mathrm{F}-\mathrm{F}_{1}} \mathbf{x}_{\mathrm{F}-\mathrm{F}_{1}} \\
+ & \boldsymbol{\Phi}_{\mathrm{F}_{1}} \mathbf{x}_{\mathrm{F}_{1}}-\boldsymbol{\Phi}_{\mathrm{F}_{1}}\left(\boldsymbol{\Phi}_{\mathrm{F}_{1}}^{\mathrm{T}} \boldsymbol{\Phi}_{\mathrm{F}_{1}}\right)^{-1} \boldsymbol{\Phi}_{\mathrm{F}_{1}}^{\mathrm{T}} \cdot \boldsymbol{\Phi}_{\mathrm{F}_{1}} \mathbf{x}_{\mathrm{F}_{1}} \\
& =\boldsymbol{\Phi}_{\mathrm{F}-\mathrm{F}_{1}} \mathbf{x}_{\mathrm{F}-\mathrm{F}_{1}}-\boldsymbol{\Phi}_{\mathrm{F}_{1}}\left(\boldsymbol{\Phi}_{\mathrm{F}_{1}}^{\mathrm{T}} \boldsymbol{\Phi}_{\mathrm{F}_{1}}\right)^{-1} \boldsymbol{\Phi}_{\mathrm{F}_{1}}^{\mathrm{T}} \cdot \boldsymbol{\Phi}_{\mathrm{F}-\mathrm{F}_{1}} \mathbf{x}_{\mathrm{F}-\mathrm{F}_{1}}
\end{aligned}
$$

Therefore

$$
\begin{gathered}
\left\|\mathbf{r}_{1}\right\|_{2}=\left\|\boldsymbol{\Phi}_{\mathrm{F}-\mathrm{F}_{1}} \mathbf{X}_{\mathrm{F}-\mathrm{F}_{1}}-\boldsymbol{\Phi}_{\mathrm{F}_{1}}\left(\boldsymbol{\Phi}_{\mathrm{F}_{1}}^{\mathrm{T}} \boldsymbol{\Phi}_{\mathrm{F}_{1}}\right)^{-1} \boldsymbol{\Phi}_{\mathrm{F}_{1}}^{\mathrm{T}} \cdot \boldsymbol{\Phi}_{\mathrm{F}-\mathrm{F}_{1}} \mathbf{x}_{\mathrm{F}-\mathrm{F}_{1}}\right\|_{2} \leq\left\|\boldsymbol{\Phi}_{\mathrm{F}-\mathrm{F}_{1}} \mathbf{x}_{\mathbf{F}-\mathbf{F}_{1}}\right\|_{2} \text { (Lemma 3) } \\
\leq \sqrt{1+\delta_{k}} \cdot c_{K}\left\|\mathbf{x}_{\mathbf{F}-\mathrm{F}_{1-1}}\right\|_{2}
\end{gathered}
$$

Similarly

$$
\begin{aligned}
\left\|\mathbf{r}_{1-1}\right\|_{2} \geq & \frac{1-\delta_{K}-\delta_{2 K}}{1-\delta_{K}}\left\|\boldsymbol{\Phi}_{\mathbf{F}-\mathbf{F}_{1-1}} \mathbf{x}_{\mathbf{F}-\mathbf{F}_{1-1}}\right\|_{2} \\
\geq & \frac{1-2 \delta_{2 K}}{1-\delta_{K}} \sqrt{1-\delta_{K}}\left\|\mathbf{x}_{\mathbf{F}-\mathbf{F}_{1-1}}\right\|_{2} \\
& \geq \frac{1-2 \delta_{2 K}}{\sqrt{1-\delta_{K}}}\left\|\mathbf{x}_{\mathbf{F}-\mathbf{F}_{1-1}}\right\|_{2}
\end{aligned}
$$

The following formula can be established based on Formulas (17) and (18):

$$
\begin{aligned}
\left\|\mathbf{r}_{1}\right\|_{2} & \leq \frac{\sqrt{1-\delta^{2}{ }_{K}}}{1-2 \delta_{2 K}} c_{K}\left\|\mathbf{r}_{1-1}\right\|_{2} \\
& \leq \frac{c_{K}}{1-2 \delta_{3 K}}\left\|\mathbf{r}_{1-1}\right\|_{2} \quad \text { (Lemma 1) }
\end{aligned}
$$


When $\delta_{3 K} \leq 0.165, \frac{c_{K}}{1-2 \delta_{3 K}}<1$, the conclusions are proved.

\section{Experiments and Simulation}

In this paper, Matlab is used to conduct the simulation experiment. The experiment could be divided into two parts: In the first part of the experiment, we used the proposed algorithm to conduct the reconstruction for the general sparse vector of artificially constructed structure, and verified the correctness of the proposed algorithm through comparing the reconstructed data and the original data. In the second part of the experiment, we used the proposed algorithm to conduct the reconstruction for the actual wireless sensor network data, compared the advantages and disadvantages between the proposed algorithm and the previous algorithms from the perspective of reconstruction accuracy, and thus verified the effectiveness of the proposed algorithm.

In order to construct a vector to be reconstructed with a length of 900 , firstly we fixed the sparsity of the sparse vector, and let the number of its non-zero elements be 20 . When we used the algorithm in this paper and the traditional algorithm to conduct the reconstruction for the sparse vector, we transformed the value of the measurement $J$ and made it increase by degrees among 1-400. We repeated the experiment 100 times for every different measurement, if the mean square error (MSE) of signal reconstruction was less than $2 e-03$, the reconstruction was considered to be successful. The success rate of the reconstruction is the ratio between the number of success and the total number of experiments. From the experimental results shown in Figure 2 we can see that, as for the coefficient vector with fixed sparsity, the algorithm in this paper could achieve the reconstruction performance which was similar to the traditional algorithms. Furthermore, because the sparsity of the vector could be estimated in the algorithm, it could be more effective to conduct the reconstruction with the measurements.

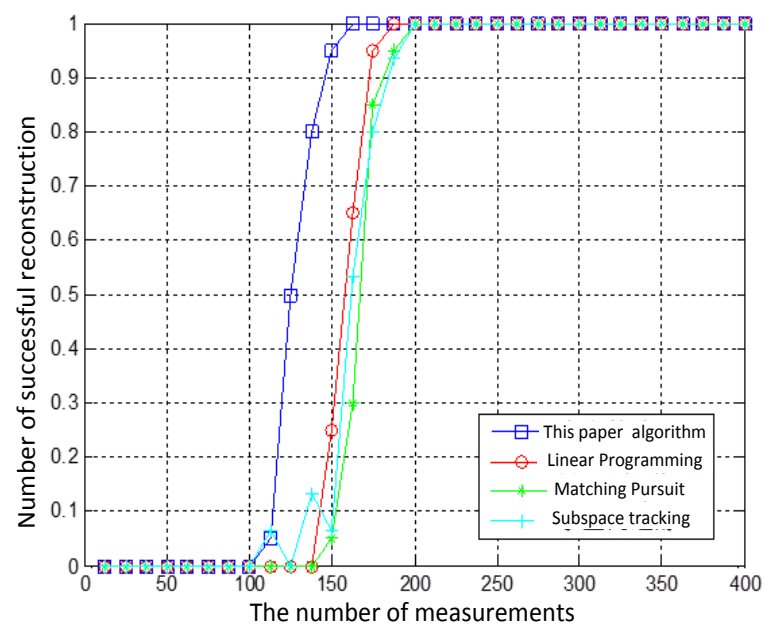

\section{Figure 1. Comparison of Reconstruction Success Rate}

In the second part of the experiment, the wireless sensor network model shown in Figure 1 was used, and there were totally 900 randomly distributed sensor nodes. After the sensor nodes conducted acquisition for the environmental temperature in the region, the data was sent to Sink nodes respectively. In order to verify the effectiveness of the algorithm in this paper in the reconstruction of weak spatial correlation network data, we used two groups of network data in the experiment, one was the original data in the ideal situation, and the other was the network data affected by noise pollution. From the original data we can see that, the network data in the ideal situation (Figure 3 (a)) had 
better spatial correlation, and thus its DCT transformation domain coefficient (Figure 4 (a)) had good sparsity; while the spatial correlation of the network data affected by noise pollution (Figure 3 (b)) was not obvious, and at the same time the sparsity of its transformation domain coefficient (Figure 4 (b)) was also poor.

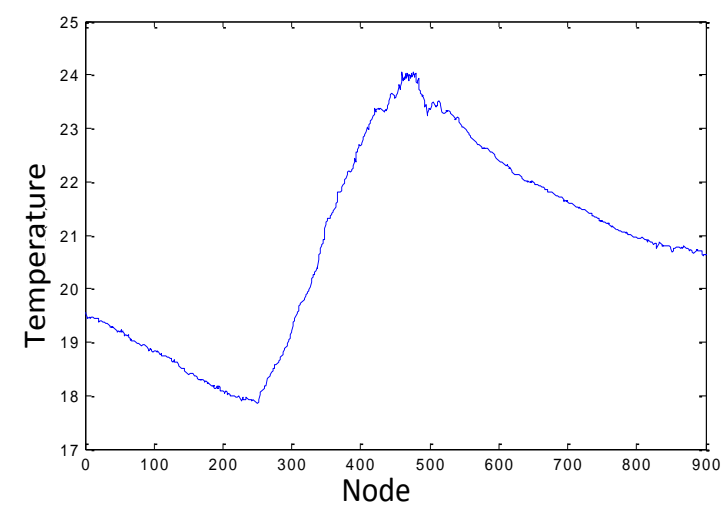

(a)

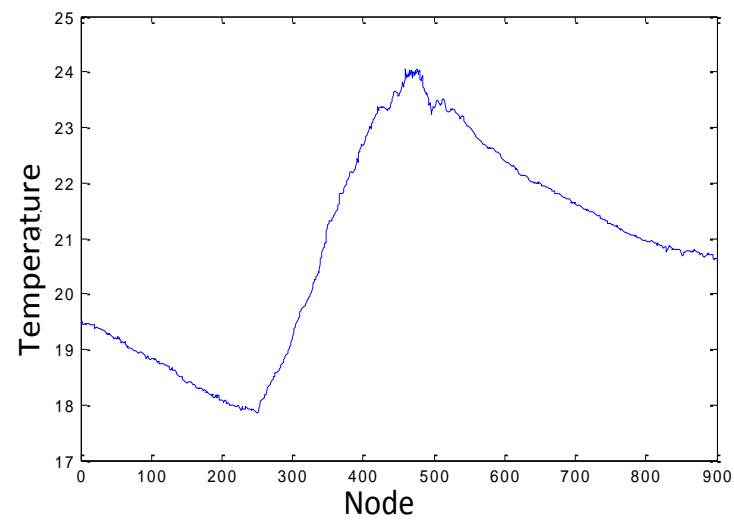

(b)

Figure 2. Original Temperature Data in Time Domain

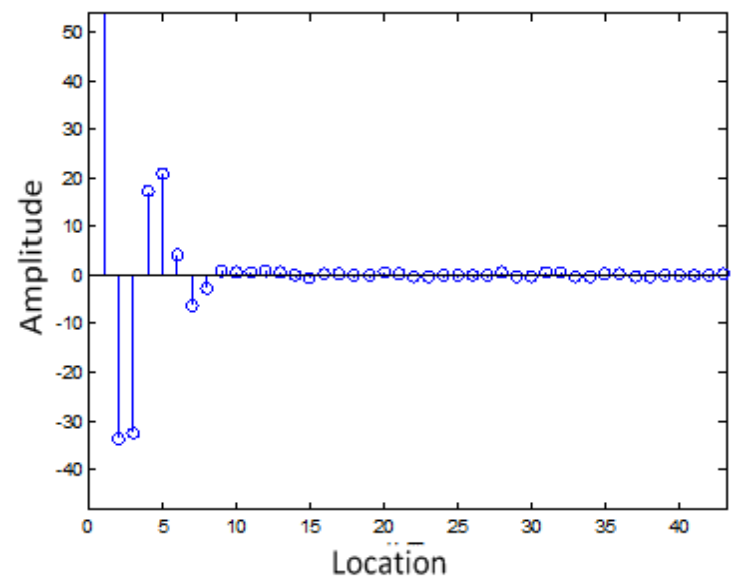

(a) 


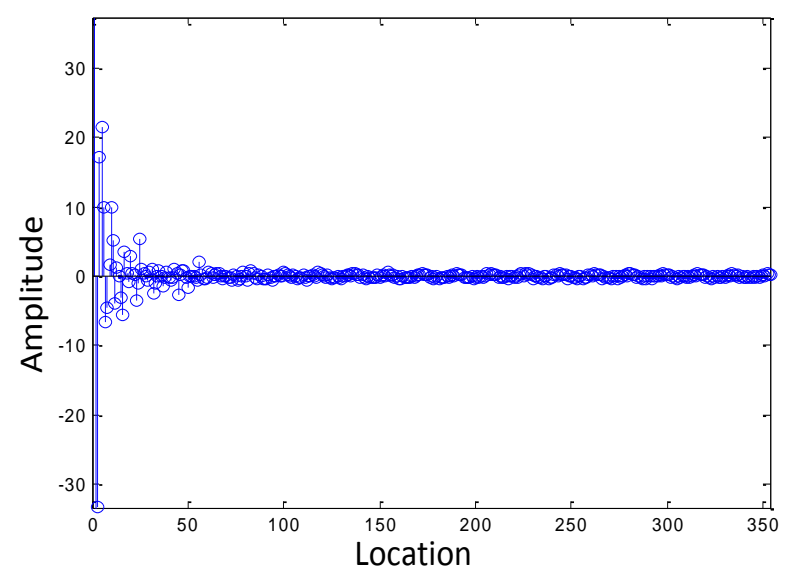

(b)

Figure 3. Transformation Domain Coefficient of Temperature Data

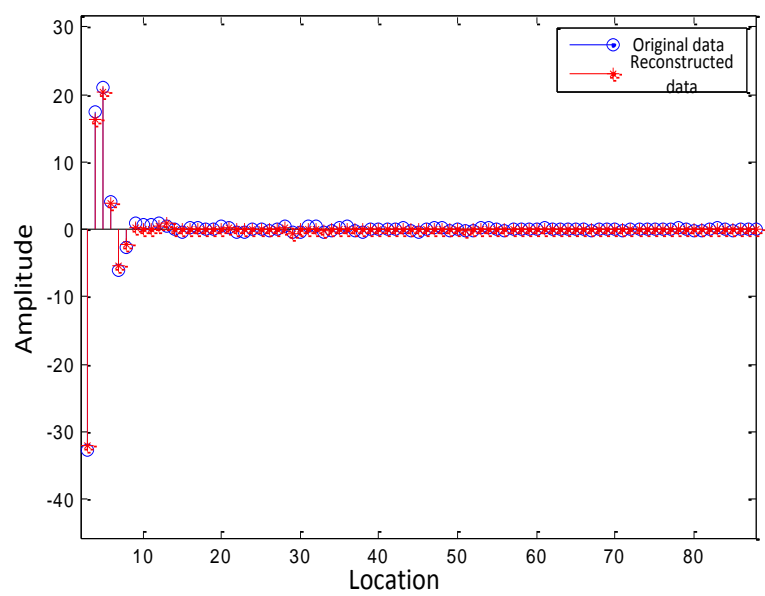

(a)

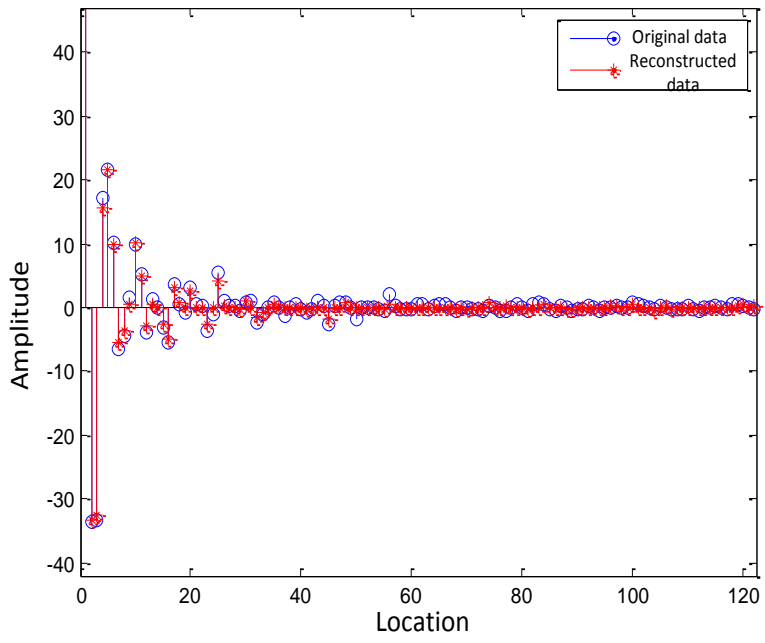

(b)

Figure 4. Comparison between Transformation Domain Reconstruction Signal and Original Signal 
In the experiment, we supposed that the pseudo-random number generated locally by each sensor obeyed Gaussian distribution. After the data perceived by the nodes were multiplied with the pseudo-random sequence, they were sent to Sink nodes. At the receiving end, the iterative convex optimization algorithm was used to reconstruct the obtained receiving data. Figure 5 is the comparison between the obtained reconstruction data and the original data when 1/6 data transmission amount is used. From the simulation results we can see that, the algorithm in this paper can conduct accurate reconstruction for the two groups of network data with different correlations, the reconstruction data is basically consistent with the original data, and there is only slight difference in amplitude.

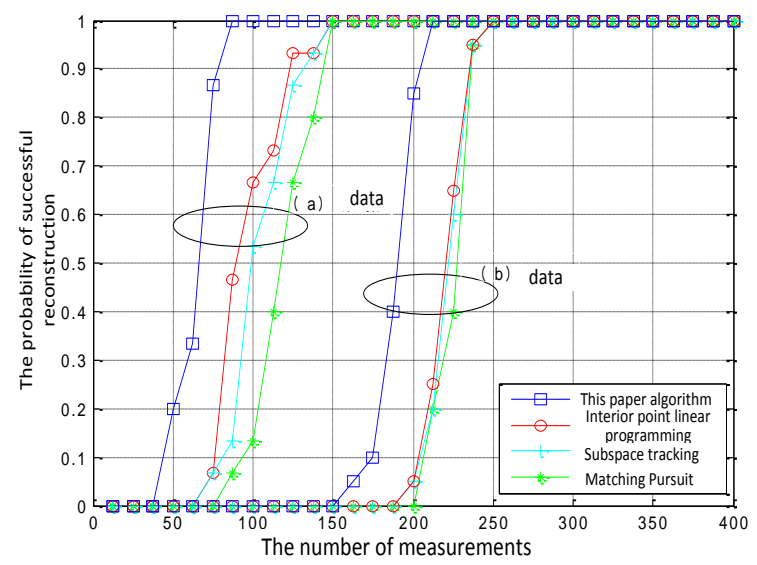

\section{Figure 5. Comparison of Reconstruction Accuracy under Different Correlations}

\section{Conclusions}

Energy constraint of sensor nodes is one of the key challenges in the technology development of wireless sensor networks, therefore, it is the key technology to effectively compress network data and reduce the energy-consumption during data transmission. Traditional reconstruction algorithm of compressive sensing in network data requires the prior information such as the correlation of networks data, while the data reconstruction of actual wireless sensor network cannot meet the requirements. The proposed correlation adaptive reconstruction algorithm can gradually estimate the correlation of network data, and adaptively use an appropriate amount of measurements for construction. On the one hand, compared to traditional distributed compressive sensing theory, the proposed algorithm improves the stability and robustness of network data reconstruction; on the other hand, it can efficiently use the measurements of network data to further reduce the measurement computing of network data.

\section{References}

[1] D. Donoho, "Compressed sensing", IEEE Trans. Information Theory, vol. 52, no. 4, (2006), pp. 1289-1306.

[2] E. Candes, "Compressive sampling", Proceedings of the International Congress of Mathematicians, Madrid, Spain, (2006).

[3] E. Candes, J. Romberg and T. Tao, "Robust uncertainty principles: Exact signal reconstruction from highly incomplete frequency information", IEEE Trans. Information Theory, vol. 52, no. 4, (2006), pp. 489-509.

[4] S. Chen, D. Donoho and M. Saunders, "Atomic Decomposition by Basis Pursuit", SIAM Journal on Scientific Computing, vol. 20, no. 1, (1999), pp.33-61.

[5] S. Kim, K. Koh and M. Lustig, "An interior-Point method for large-scale 11 regularized least squares", IEEE Journal of Selected Topics in Signal processing, vol. 1, no. 4, (2007), pp. 606-617. 
[6] J. Tropp and A. Gilbert, "Signal recovery from random measurements via orthogonal matching pursuit", IEEE Trans. Info. Theory, vol. 53, (2007), pp. 4655-4666.

[7] D. L. Donoho, Y. Tsaig and J.-L. Starck, "Sparse solution of underdetermined linear equations by stagewise orthogonal matching pursuit", Technical Report, (2006).

[8] W. Dai and O. Milenkovic, "Subspace pursuit for compressive sensing", Closing the gap between performance and complexity, IEEE Trans. Info. Theory, vol. 55, no. 5, (2009), pp. 2230-2249.

[9] D. Needell and J. A. Tropp, "Cosamp: Iterative signal recovery from incomplete and inaccurate samples", Preprint, Mar 2008. Applied and Computational Harmonic Analysis, vol. 26, no. 3, (2009), pp. 301-321.

[10] J.-A.M. Mondol, "Cloud security solutions using FPGA", In Communications, Computers and Signal Processing (PacRim), 2011 IEEE Pacific Rim Conference on, (2011).

[11] W. Lina, G. Hanjn, Liuwei and P. Yang, "Detection and management of virtual machine monitor", Research and development process of Computer, (2011), pp. 1534-1541.

\section{Author}

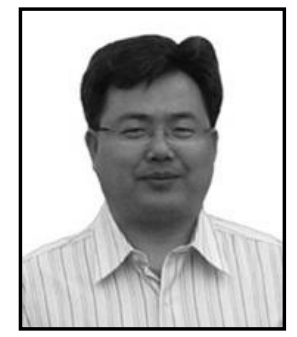

Wang Changliang, he was born in 1973, in Shanxi province. He received his degree in Southeast University. His research interests are networks analysis and data mining. 